\title{
Effects of Finite Rate Heterogeneous Kinetics on Homogeneous Ignition in Catalytically Stabilized Channel Flow Combustion
}

\author{
JOHN MANTZARAS* and CHRISTOPH APPEL \\ Paul Scherrer Institute, Combustion Research, CH-5232 Villigen-PSI, Switzerland
}

\begin{abstract}
The homogeneous (gas-phase) and heterogeneous (catalytic) ignition of fuel-lean premixtures is investigated analytically and numerically in two-dimensional laminar channel-flow configurations with uniform incoming properties and isothermal catalytic walls. First-order matched activation energy asymptotics are employed, along with a one-step catalytic reaction and a one-step large activation energy gaseous reaction. Parametric description of the chemically frozen state leads to a closed-form heterogeneous ignition criterion in terms of non-dimensional variables that are relevant to confined flows. Formulation of the weakly reactive state yields a closed-form homogeneous ignition criterion that includes explicitly the homogeneous and heterogeneous reactivities through the relevant gaseous and surface Damköhler numbers ( $D a_{g}$ and $D a_{s}$, respectively). Both ignition criteria are valid over the range $0.002 \leq x /(b R e P r) \leq 0.16,1.5 \leq T_{W} / T_{I N} \leq 3$, and $0.9 \leq L e \leq 2.0$, where $x$ is the streamwise distance, $R e$ the incoming Reynolds number based on the channel half-height $b, P r$ the Prandtl number, $T_{W} / T_{I N}$ the wall-to-inlet temperature ratio, and $L e$ the Lewis number of the fuel. Numerical simulations have shown good agreement between the numerically and the analytically predicted homogeneous ignition distances. A reduction of the surface reactivity $\left(D a_{s}\right)$ promotes homogeneous ignition due to the associated increase of the near-wall fuel levels, and this effect is manifested in the homogeneous ignition criterion via a corresponding increase of the characteristic transverse diffusion time scale with decreasing $D a_{s}$. It is shown that there exist infinite combinations of surface and gaseous reactivities yielding the same homogeneous ignition distance, suggesting caution in the interpretation of catalytically stabilized combustion (CST) experiments. Moreover, the homogeneous ignition distance is much more sensitive to the gaseous rather than to the surface reaction pathway, thus exemplifying the importance of validated homogeneous reaction schemes under CST-relevant conditions. (C) 2002 by The Combustion Institute
\end{abstract}

\section{NOMENCLATURE}

$A$

$b$

$B_{s}, B_{g}$

$\tilde{B}$

$c_{p}$

$D_{i}$

$D a_{s}, D a_{g}$

$E_{s}, E_{g}$

$f$

$F$

G

*Corresponding author. E-mail: ioannis.mantzaras@psi.ch

40

Channel half-height

Frequency factors of surface and gaseous reactions, Eqs. 2 and 3

Function defined in Eq. A3

Specific heat at constant

pressure

Species diffusivity

Surface and gas-phase

Damköhler numbers, Eqs. 21 and 46

Activation energies of surface and gaseous reactions, Eqs. 2 and 3

Non-dimensional stream

function, Eq. 14

Function defined in Eq. 41

Function defined in Eq. 30

$0010-2180 / 02 / \$-$ see front matter

PII S0010-2180(02)00384-X
$H$

$I_{h, F}$

Function defined in Eq. 42

Fractional catalytic fuel conversion, Eq. 37

Le $\quad$ Lewis number $(=S c / P r)$

$\dot{m}_{h, F}, \dot{m}_{F, I N} \quad$ Catalytic fuel conversion and inlet fuel flow rate, Eqs. 35 and 36

$n_{i}, n \quad$ Reaction order $(i=F, O)$, sum of reaction orders, Eq. A1

$p, p^{*} \quad$ Pressure, non-dimensional pressure

$P$

$\mathrm{Pr}$

$q$

$Q$

$r$

Function defined in Eq. 34

Prandtl number $\left(=c_{p} \mu / \lambda\right)$

Heat of combustion per unit mass of fuel

Function defined in Eq. A11

Sign-determining exponent in

Eqs. 22 and 23

$R \quad$ Universal gas constant

Re Reynolds number, Eq. 13

$s \quad$ Dimensionless streamwise distance, Eq. 12

$\dot{S}_{i} \quad$ Catalytic reaction rate of species $i$, Eq. 2

COMBUSTION AND FLAME 130:336-351 (2002)

(C) 2002 by The Combustion Institute

Published by Elsevier Science Inc. 


$S c$
$T, \tilde{T}$
$\tilde{T}_{a}$
$U_{I N}$
$u, v$
$W_{\dot{v}} \bar{W}$
$x, y$
$Y_{\dot{v}} \tilde{Y}_{i}$

\section{Greek Symbols}

$\alpha_{t h} \quad$ Thermal diffusivity $(=\mu / \rho P r)$

$\alpha \quad$ Parameter defined in Eq. A14

$\beta \quad$ Parameter defined in Eq. A1

$\gamma \quad$ Gaseous reaction temperature exponent, Eq. 3

$\Delta_{c n} \Delta_{c r}^{*} \quad$ Critical and critical ignition Damköhler numbers, Eq. A16

$\epsilon \quad$ Small perturbation parameter, Eq. A6

$\zeta \quad$ Non-dimensional streamwise distance, Eq. 27

$\eta, \eta^{*} \quad$ Transverse coordinate (Eq. 12), distance at symmetry plane $(y=b)$

$\theta$

$\lambda$

$\mu$

$\nu_{i}$

$\xi$

$\rho \quad$ Density

$\tau_{g, c h} \quad$ Characteristic gaseous chemical time, Eq. 44

$\tau_{d} \quad$ Characteristic transverse diffusion time, Eq. 45

$\tau_{\text {eff, } d} \quad$ Effective transverse diffusion time, Eq. 48

$\phi, \varphi \quad$ Equivalence ratio, perturbation parameter for $\theta$ $\psi$

$\omega_{i}$

$\tilde{\omega}$

\section{Subscripts}

$F, O, P$
$f r$
$i$
$i g$
IN
$i n r$
$s, g$
$W$
$\chi$

Stream function or species perturbation parameter, Eq. A7 Species reaction rate, Eq. 16 Non-dimensional reaction parameter, Eq. A2

\author{
Fuel, Oxidizer, Product \\ Chemically frozen \\ Index for species \\ Ignition \\ Inlet \\ Inner solution \\ Surface, gas \\ Wall \\ Differentiation with respect to $\chi$
}

\section{Superscripts}

Differentiation with respect to $\eta$

\section{INTRODUCTION}

Catalytically stabilized combustion (CST) provides the best available low-NOx combustion technology for gas turbines (demonstrated NOx emissions less than $3 \mathrm{ppm}$ in small-scale machines [1]), with the added advantage of significant cost reductions compared to competing NOx-aftertreatment techniques such as selective catalytic reduction (SCR) or NOx-storage reduction (SCONOx) [2]. The increased temperature requirements of current generation turbines (up to $1780 \mathrm{~K}$ ) and the catalyst/substrate thermal stability limitations (acceptable temperatures less than $1400 \mathrm{~K}$ ) restrict the amount of heterogeneous (catalytic) fuel conversion to about $50 \%$. The catalytic processes, in turn, stabilize via thermal and chemical interactions a subsequent homogeneous (gas-phase) combustion zone, where complete conversion of the remaining fuel and the unburned $\mathrm{CO}$ is achieved.

Key issues in CST advancement are the development of ignition catalysts active enough at the relatively low compressor discharge temperatures (ca. $700 \mathrm{~K}$ ) and the very high spatial velocities of gas turbines, and the attainment of long-term catalyst thermal stability. The former requirement is controlled largely by the physi- 
cochemical properties of the fuel/catalyst system, and current research efforts focus on $\mathrm{Pd} /$ PdO-based ignition catalysts [3]. Long-term catalyst stability, on the other hand, is controlled primarily by reactor design and not by physicochemical properties. Catalyst operation at the corresponding fuel/air adiabatic flame temperature (corrected appropriately for diffusionally imbalanced fuels [4]) presents an unattainable upper-limit, notwithstanding recent advances in mid- or high-temperature catalyst technology [5]; thermal stability considerations require operation at lower temperatures, and this is achieved with design solutions such as alternate catalytically active/inactive channel coatings [6], diffusional barrier layers [7] for fuels with Lewis number $L e \leq 1$ (e.g., methane), etc. Crucial issues in catalytic reactor design are the attainment of the maximum possible fuel conversion with acceptable pressure drop and without catalyst overheat or initiation of gaseous combustion within the catalytic reactor; the latter event, in particular, can lead to catalyst meltdown.

The delineation of safe operating conditions that ensure no homogeneous ignition inside the catalyst is, therefore, of prime interest in CST. Such information requires the knowledge of validated hetero/homogeneous chemical reaction schemes, the development of multidimensional numerical models incorporating detailed chemistry, and the identification of the key parameters controlling homogeneous ignition. Elementary heterogeneous reaction schemes for the oxidation of simple fuels over Pt and Pd have advanced significantly over the last years [8-11] complementing the extensive literature on gas-phase schemes, and multidimensional CST codes are available nowadays [12, 13]. Furthermore, the validation of various hetero/ homogeneous reaction schemes in their capacity to reproduce measured homogeneous ignition characteristics was initiated recently [14, 15]. Analytical homogeneous ignition criteria for channel-flow CST were developed in Mantzaras and Benz [16] using matched activation energy asymptotics, an infinitely-fast catalytic reaction (mass-transport-limited operation), a single-step large activation energy gaseous reaction, and isothermal wall boundary conditions. These criteria were further adapted to atmospheric-pressure $\mathrm{CH}_{4}$ /air CST [17], providing homogeneous ignition distances in good agreement to those predicted numerically using the hetero/homogeneous reaction schemes that were validated in Dogwiler et al. [14]. The aforesaid good agreement is attributed to the fact that, in atmospheric-pressure $\mathrm{CH}_{4}$ /air CST, homogeneous ignition and finite-rate catalytic kinetics are, largely, mutually exclusive events: homogeneous ignition of a hard-to-ignite fuel such as methane requires sufficiently high catalyst and/or inlet temperatures that, in turn, force catalytic operation close to the mass-transport limit.

Finite-rate surface chemistry is, nevertheless, of particular importance under realistic conditions. High-pressure operation exacerbates homogeneous ignition because of the corresponding increase of the gas-phase reactivity of hydrocarbons [18]; homogeneous ignition can, therefore, occur at considerably lower temperatures and therein finite-rate surface kinetics could come to importance. In addition, many combustor designs such as alternate catalytically active/inactive (or active/partially active) coatings, warrant finite-rate surface kinetics on the partially active (or even on the room-temperature inactive) surfaces; finite-rate surface kinetics can, in turn, promote homogeneous ignition due to the associated near-wall fuel excess. Analytical homogeneous ignition studies in catalytic combustion have been reported for external flows, for example, stagnation point flows [19-21] or flat-plate flows [22, 23], and more recently for confined flows [16]. Finite rate surface kinetics were accounted for in the 1-D stagnation-point flow studies [19-21], whereas in the 2-D cases an infinitely-fast surface reaction was considered [22, 23, 16]. Analytical studies in 2-D channel-flow configurations relevant to CST with combined finite-rate surface and gaseous kinetics have not been reported. The present study undertakes an analytical and numerical investigation of isothermal-wall channel CST with main objective to provide closedform homogeneous ignition criteria that include explicitly the effect of finite-rate surface chemistry. The analysis is based on first order matched activation energy asymptotics in conjunction with the boundary layer approximation, a one-step surface reaction, and a one-step 


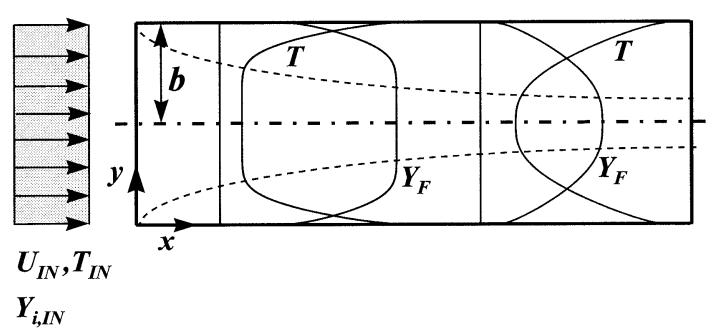

Fig. 1. Plane channel-flow catalytic configuration with uniform inlet properties. Typical transverse $(y)$ profiles of the fuel mass fraction $\left(Y_{F}\right)$ and temperature $(T)$ under finiterate surface chemistry conditions. The dotted lines mark the extent of the thermal boundary layer.

large activation energy gaseous reaction. $\mathrm{Nu}-$ merical computations are also performed-using the same underlying assumptions as in the analytical work-in order to assess the range of applicability of the analytical solution. Particular objectives are to further establish catalytic ignition criteria for channel-flow CST, and to quantify the sensitivity of homogeneous ignition on both the heterogeneous and the homogeneous reaction pathways; the latter is of particular importance for the validation of hetero/ homogeneous reaction schemes with homogeneous ignition investigations $[14,15]$.

This article is organized as follows. First the mathematical model is given and the parametric description of the chemically frozen state is presented for a first-order (with respect to the deficient reactant) catalytic reaction. The chemically frozen state leads naturally to a heterogeneous ignition criterion. Formulation of the weakly reactive state leads to the corresponding homogeneous ignition criteria. Comparisons between analytically and numerically predicted homogeneous ignition distances follow and, finally, the sensitivity of homogeneous ignition on both reaction pathways is assessed.

\section{MATHEMATICAL FORMULATION}

A plane channel configuration with uniform incoming flow properties is considered. It consists of two parallel catalytic plates at a distance $2 b$ apart (see Fig. 1), which are maintained at a fixed temperature $T_{W}$. A single-step irreversible Arrhenius reaction is considered for both surface and gaseous pathways:

$$
[F]+\nu_{O}[O] \rightarrow \nu_{P}[P] .
$$

To facilitate the ensuing parametric description of the chemically frozen state, a catalytic reaction first-order with respect to the fuel and zero-order with respect to the oxidizer is considered. The surface fuel reaction rate, $\dot{S}_{F}$, can be expressed as:

$\dot{S}_{F}=W_{F} B_{s}\left(\frac{\rho Y_{F}}{W_{F}}\right)_{W} \exp \left(-E_{s} / R T_{W}\right)$.

The surface rate assumptions are not restrictive for CST since hydrocarbons exhibit first order (with respect to the fuel) global kinetics on precious metal surfaces [24-26].

The gaseous reaction rate has no other restrictions apart from that of a large activation energy. The volumetric gaseous fuel reaction rate, $\dot{\omega}_{F}$, is:

$\dot{\omega}_{F}=W_{F} B_{g} T^{\gamma}\left(\frac{\rho Y_{F}}{W_{F}}\right)^{n_{F}}\left(\frac{\rho Y_{O}}{W_{O}}\right)^{n_{O}} \exp \left(-E_{g} / R T\right)$.

Finite-rate surface kinetics are manifested by the non-zero wall concentration of the deficient reactant (fuel). In the entry flow configuration of Fig. 1, finite-rate surface kinetic effects (increased fuel wall concentrations) are more pronounced at shorter streamwise distances. Under the boundary layer approximation, the governing equations become [27]:

$\frac{\partial(\rho u)}{\partial x}+\frac{\partial(\rho v)}{\partial y}=0$,

$\rho u \frac{\partial u}{\partial x}+\rho v \frac{\partial u}{\partial y}-\frac{\partial}{\partial y}\left(\mu \frac{\partial u}{\partial y}\right)=-\frac{d p}{d x}$,

$\rho u c_{p} \frac{\partial T}{\partial x}+\rho v c_{p} \frac{\partial T}{\partial y}-\frac{\partial}{\partial y}\left(\lambda \frac{\partial T}{\partial y}\right)=q \dot{\omega}_{F}$,

$\rho u \frac{\partial Y_{i}}{\partial x}+\rho v \frac{\partial Y_{i}}{\partial y}-\frac{\partial}{\partial y}\left(\rho D_{i} \frac{\partial Y_{i}}{\partial y}\right)=-\dot{\omega}_{i}$,

respectively, with $\partial p / \partial y=0$. The system of equations is closed with the ideal gas law,

$p=\rho \frac{R}{\bar{W}} T$.

The set of Eqs. 4 through 8 constitutes a parabolic system with initial conditions $(x=0)$ : 


$$
\begin{aligned}
u=U_{I N}, \quad v & =0, \quad T=T_{I N}, \quad p=p_{I N} \\
\text { and } \quad Y_{i} & =Y_{i, I N} .
\end{aligned}
$$

The boundary conditions at the gas-wall interface $(x>0, y=0)$ are:

$$
\begin{aligned}
& u=v=0, \quad T=T_{W}, \\
& \text { and }\left(\rho D_{i} \frac{\partial Y_{i}}{\partial y}\right)_{W}=\dot{S}_{i} .
\end{aligned}
$$

Finally, at the symmetry-plane $(x>0, y=b)$ the boundary conditions are:

$\frac{\partial u}{\partial y}=v=0, \quad \frac{\partial T}{\partial y}=0, \quad$ and $\quad \frac{\partial Y_{i}}{\partial y}=0$.

Relevant to confined flows are the non-dimensional streamwise and transverse coordinates

$s=x /(b R e)$ and $\eta=\frac{1}{b \rho_{I N} \sqrt{2 s}} \int_{0}^{y} \rho d y$,

respectively [16], with $R e$ the inlet Reynolds number based on the channel half-height:

$R e=\frac{\rho_{I N} U_{I N} b}{\mu_{I N}}$.

Introducing the stream function $\psi(x, y)$ and the non-dimensional dependent variables,

$f=\frac{\psi}{b \rho_{I N} U_{I N} \sqrt{2 s}}, \quad p^{*}=\frac{p}{\rho_{I N} U_{I N}^{2}}$,

$\theta=\frac{T_{W}-T}{T_{W}-T_{I N}}, \quad \tilde{Y}_{i}=\left(\frac{W_{F}}{\nu_{i} W_{i}}\right) Y_{i}$

and assuming equal species diffusivities $D_{i}=D$, constant values for $\rho \mu, \rho \lambda, \rho^{2} D$, and $c_{p}$ (resulting in constant Prandtl and Schmidt numbers), the governing Eqs. 4 through 8 become:

$$
\begin{aligned}
\frac{\partial^{3} f}{\partial \eta^{3}} & +f \frac{\partial^{2} f}{\partial \eta^{2}}+2 s\left(\frac{\partial f}{\partial s}\right) \frac{\partial^{2} f}{\partial \eta^{2}}-2 s\left(\frac{\partial f}{\partial \eta}\right) \frac{\partial^{2} f}{\partial \eta \partial s} \\
& =2 s\left(\frac{\rho_{I N}}{\rho}\right)\left(\frac{d p^{*}}{d s}\right) \\
\operatorname{Pr}^{-1} & \frac{\partial^{2} \theta}{\partial \eta^{2}}+f \frac{\partial \theta}{\partial \eta}+2 s\left(\frac{\partial f}{\partial s}\right) \frac{\partial \theta}{\partial \eta}-2 s\left(\frac{\partial f}{\partial \eta}\right) \frac{\partial \theta}{\partial s} \\
= & 2 s \frac{R e b q}{U_{I N} c_{p}\left(T_{W}-T_{I N}\right)}\left(\frac{\dot{\omega}_{F}}{\rho}\right)
\end{aligned}
$$

$$
\begin{aligned}
& S c^{-1} \frac{\partial^{2} \tilde{Y}_{i}}{\partial \eta^{2}}+f \frac{\partial \tilde{Y}_{i}}{\partial \eta}+2 s\left(\frac{\partial f}{\partial s}\right) \frac{\partial \tilde{Y}_{i}}{\partial \eta}-2 s\left(\frac{\partial f}{\partial \eta}\right) \frac{\partial \tilde{Y}_{i}}{\partial s} \\
& =2 s \frac{\operatorname{Reb}}{U_{I N}}\left(\frac{\dot{\omega}_{F}}{\rho}\right) \\
& p=\rho \frac{R}{\bar{W}}\left[T_{W}-\theta\left(T_{W}-T_{I N}\right)\right] .
\end{aligned}
$$

The initial conditions $(s=0)$ are:

$f^{\prime}=1, \quad \theta=1, \quad p^{*}=p_{I N}^{*}, \quad \frac{1}{\tilde{Y}_{i, I N}} \tilde{Y}_{i}=1$.

Using Eqs. 12, 14, and $\rho_{I N}^{2} D_{I N}=\rho_{W}^{2} D_{W}$, the interfacial $(s>0, \eta=0)$ boundary conditions of Eqs. 10 become:

$f=0, \quad f^{\prime}=0, \quad \theta=0$,

and

$\frac{1}{\tilde{Y}_{F, I N}}\left(\frac{\partial \tilde{Y}_{F}}{\partial \eta}\right)_{W}=L e \sqrt{2 s}\left(\frac{\rho_{W}}{\rho_{I N}}\right)\left(\frac{\tilde{Y}_{F, W}}{\tilde{Y}_{F, I N}}\right) D a_{s}$,

with $D a_{s}$ a characteristic surface Damköhler number,

$D a_{s}=\frac{b B_{s} \exp \left(-E_{s} / R T_{W}\right)}{\alpha_{t h, I N}}$.

Given the equal species diffusivity assumption, the interfacial boundary conditions for species other than fuel are:

$$
\left(\frac{\partial \tilde{Y}_{i}}{\partial \eta}\right)_{W}=(-1)^{r}\left(\frac{\partial \tilde{Y}_{F}}{\partial \eta}\right)_{W},
$$

with $r=0$ for the oxidizer and $r=1$ for the products. Equations 22, under the equal diffusivity assumption, are equivalent to:

$$
\left(\tilde{Y}_{F, W}-\tilde{Y}_{F, I N}\right)=(-1)^{r}\left(\tilde{Y}_{i, W}-\tilde{Y}_{i, I N}\right) .
$$

Finally, at the plane-of-symmetry $(s>0, \eta=$ $\left.\eta^{*}\right)$ the boundary conditions become:

$f^{\prime \prime}=0, \quad \theta^{\prime}=0, \quad \tilde{Y}_{i}^{\prime}=0$.

For the high inlet Reynolds numbers of CST applications, the boundary layer approximation is valid for purely catalytic combustion [28]; for gaseous combustion it is valid at sufficiently high inlet velocities or low fuel-to-air equivalence ratios [17]. 


\section{CHEMICALLY FROZEN STATE}

Before homogeneous ignition the gas phase is chemically frozen $\left(E_{g} / R T_{W} \rightarrow \infty\right)$ and fuel is converted only heterogeneously. Setting $\dot{\omega}_{i}=0$, Eqs. 16 and 17 become:

$$
\begin{aligned}
& \operatorname{Pr}^{-1} \frac{\partial^{2} \theta}{\partial \eta^{2}}+f \frac{\partial \theta}{\partial \eta}+2 s\left(\frac{\partial f}{\partial s}\right) \frac{\partial \theta}{\partial \eta}-2 s\left(\frac{\partial f}{\partial \eta}\right) \frac{\partial \theta}{\partial s} \\
& =0, \text { and } \\
& S c^{-1} \frac{\partial^{2} \tilde{Y}_{i}}{\partial \eta^{2}}+f \frac{\partial \tilde{Y}_{i}}{\partial \eta}+2 s\left(\frac{\partial f}{\partial s}\right) \frac{\partial \tilde{Y}_{i}}{\partial \eta}-2 s\left(\frac{\partial f}{\partial \eta}\right) \frac{\partial \tilde{Y}_{i}}{\partial s} \\
& =0 .
\end{aligned}
$$

The chemically frozen state is formulated by Eqs. 15, 25, 26, and 18, with the initial and boundary conditions given by Eqs. 19, 20, 22, and 24. The set of equations is intrinsically non-similar, necessitating numerical integration with a marching parabolic solver: the numerical procedure is the same as in our previous work [16] and is not repeated. An example of the chemically frozen state is illustrated in Fig. 2 for a propane/air mixture with the global catalytic reaction $\mathrm{C}_{3} \mathrm{H}_{8}+5 \mathrm{O}_{2} \rightarrow 3 \mathrm{CO}_{2}+4 \mathrm{H}_{2} \mathrm{O}$.

Parametric description of the chemically frozen state is sought in terms of the controlling geometrical, flow, transport, and surface-chemistry variables. The quantities necessary for the homogeneous and heterogeneous ignition analysis are the local transverse wall gradients of the temperature and fuel, $\theta_{f r, W}^{\prime}$ and $\left(1 / \tilde{Y}_{F, I N}\right)\left(\tilde{Y}_{F^{-}}^{\prime}\right.$ )$_{f r, W}$, respectively, and the local fuel wall concentration $\left(1 / \tilde{Y}_{F, I N}\right)\left(\tilde{Y}_{F}\right)_{f r, W}$. Closed-form expressions for the aforementioned quantities are presented in terms of a new streamwise coordinate,

$\zeta=s / \operatorname{Pr}=x /(b \operatorname{RePr})$,

which is the inverse of the Graetz number [29]. Computations of the chemically frozen state have recovered the same expression for $\theta_{f r, W}^{\prime}$ to that of our earlier, mass-transport-limited analysis [16]. $\theta_{f r, W}^{\prime}$ is a sole function of $\zeta, \operatorname{Pr}$ and $T_{W} / T_{I N}$, and is independent of $D a_{s}$ :

$$
\begin{aligned}
\theta_{f r, W}^{\prime}= & \left(0.43+0.45 \zeta^{0.35}\right) \operatorname{Pr}^{1 / 3+0.242 \zeta^{0.2}} \\
& \cdot\left(\frac{T_{W}}{T_{I N}}\right)^{0.774 \zeta^{0.2}} \cdot
\end{aligned}
$$
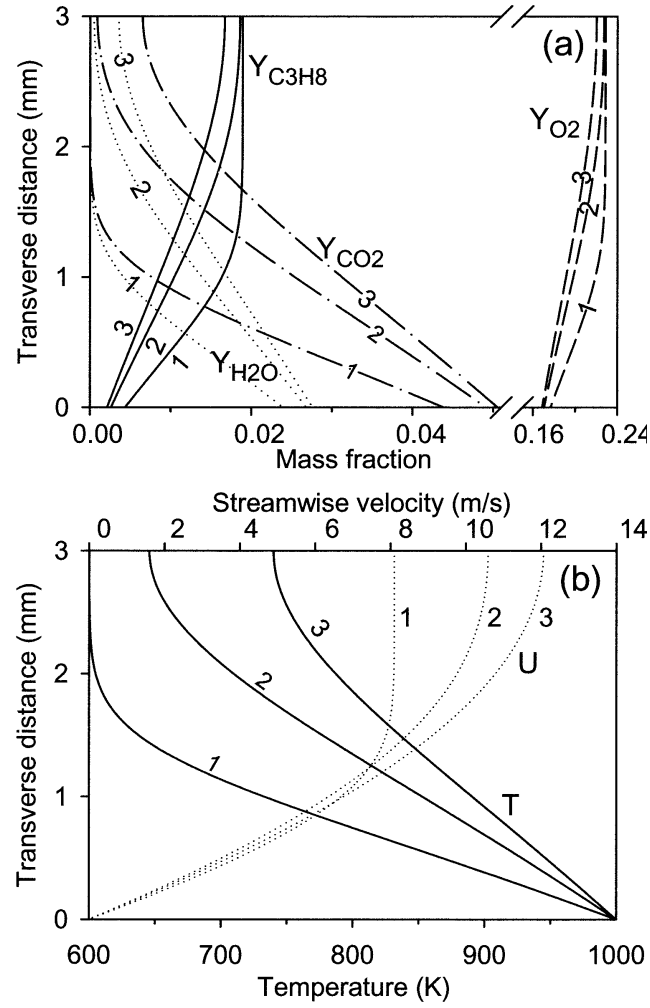

Fig. 2. Computed transverse profiles of the species mass fractions, temperature, and axial velocity at streamwise distances of $x=19.2 \mathrm{~mm}(1), x=96 \mathrm{~mm} \mathrm{(2),} \mathrm{and} x=192$ mm (3). (a): $\mathrm{C}_{3} \mathrm{H}_{8}$ (solid lines), $\mathrm{H}_{2} \mathrm{O}$ (dotted lines), $\mathrm{CO}_{2}$ (dashed-dotted lines), and $\mathrm{O}_{2}$ (dashed lines), (b): temperature (solid lines) and axial velocity (dotted lines). Pure catalytic conversion (gas-phase chemically frozen state) with $\varphi=0.30$ (propane/air mixture), $T_{I N}=600 \mathrm{~K}, T_{W}=1000$ $\mathrm{K}, U_{I N}=6 \mathrm{~m} / \mathrm{s}, p=1$ bar, $D a_{s}=13.2$, and channel half-height $b=3 \mathrm{~mm}$.

Under the infinitely-fast surface chemistry assumption, the fuel wall gradient was shown [16] to be a function of $\zeta, P r, L e$, and $T_{W} / T_{I N}$ :

$$
\left[\left(1 / \tilde{Y}_{F, I N}\right)\left(\tilde{Y}_{F}^{\prime}\right)_{f r, W}\right]_{D a_{s} \rightarrow \infty}=L e^{1 / 3+0.19 \zeta^{0.35}} \theta_{f r, W}^{\prime} .
$$

Equations 28 and 29 are valid over the range $0.002 \leq \zeta \leq 0.16,1.5 \leq T_{W} / T_{I N} \leq 3.0,0.9 \leq$ $L e \leq 2.0$ and $0.5 \leq \operatorname{Pr} \leq 1.0$ with accuracy better than $1.6 \%$ and $2.5 \%$, respectively.

Inspection of the governing equations and boundary conditions of the chemically frozen state suggests that $\left(1 / \tilde{Y}_{F, I N}\right)\left(\tilde{Y}_{F}^{\prime}\right)_{f r, W}$ and $(1 /$ $\left.\tilde{Y}_{F, I N}\right)\left(\tilde{Y}_{F}\right)_{f r, W}$ are functions of the variables $\zeta$, $\operatorname{Pr}$, Le,$T_{W} / T_{I N}$, and $D a_{s}$. Defining the function $G$, 
$G=L e \sqrt{2 \zeta \operatorname{Pr}}\left(\frac{T_{W}}{T_{I N}}\right)^{-1}$,

using Eqs. 8 and 27, and further considering constant $\bar{W}$ and $p$ (the pressure drop is small for $\zeta \leq 0.16[29,16])$, the interfacial fuel boundary condition of Eq. 20 can be written as,

$\left(1 / \tilde{Y}_{F, I N}\right)\left(\tilde{Y}_{F}^{\prime}\right)_{f r, W}=\left(1 / \tilde{Y}_{F, I N}\right)\left(\tilde{Y}_{F}\right)_{f r, W} G D a_{s}$.

Equation 31 links the wall gradient and concentration of the fuel through $G$ and $D a_{s}$, which depend solely on the proposed variables of the parametric description; this link is possible only for first-order reactions. Solutions with the following functional dependence are sought:

$$
\begin{gathered}
\left(1 / \tilde{Y}_{F, I N}\right)\left(\tilde{Y}_{F}^{\prime}\right)_{f r, W}=\left[\left(1 / \tilde{Y}_{F, I N}\right)\left(\tilde{Y}_{F}^{\prime}\right)_{f r, W}\right]_{D a_{s} \rightarrow \infty} \\
\cdot\left[\frac{G P D a_{s}}{G P D a_{s}+\left[\left(1 / \tilde{Y}_{F, I N}\right)\left(\tilde{Y}_{F}^{\prime}\right)_{f r, W}\right]_{D a_{s} \rightarrow \infty}}\right]
\end{gathered}
$$

and,

$$
\begin{gathered}
\left(1 / \tilde{Y}_{F, I N}\right)\left(\tilde{Y}_{F}\right)_{f r, W}=\left[\left(1 / \tilde{Y}_{F, I N}\right)\left(\tilde{Y}_{F}^{\prime}\right)_{f r, W}\right]_{D a_{s} \rightarrow \infty} \\
\cdot\left[\frac{P}{G P D a_{s}+\left[\left(1 / \tilde{Y}_{F, I N}\right)\left(\tilde{Y}_{F}^{\prime}\right)_{f r, W}\right]_{D a_{s} \rightarrow \infty}}\right],
\end{gathered}
$$

where the function $P$ has to be defined. The fuel wall gradient (Eq. 32) and wall concentration (Eq. 33) expressions satisfy automatically the interfacial boundary condition of Eq. 31. In addition, Eqs. 32 and 33 satisfy the following limiting conditions, provided that $P \rightarrow 1$ as $D a_{s}$ $\rightarrow 0$ and $P \cdot D a_{s} \rightarrow \infty$ as $D a_{s} \rightarrow \infty$. In the limit $D a_{s} \rightarrow \infty$, Eqs. 32 and 33 reduce to $\left(1 / \tilde{Y}_{F, I N}\right)$ $\left(\tilde{Y}_{F}^{\prime}\right)_{f r, W}=\left[\left(1 / \tilde{Y}_{F, I N}\right)\left(\tilde{Y}_{F}^{\prime}\right)_{f r, W}\right]_{D a_{s} \rightarrow \infty}$ and $(1 /$ $\left.\tilde{Y}_{F, I N}\right)\left(\tilde{Y}_{F}\right)_{f r, W}=0$, respectively, that is, to the mass-transport-limited solution; in the limit $D a_{s}$ $\rightarrow 0$, they revert to the chemically inert solution $\left(1 / \tilde{Y}_{F, I N}\right)\left(\tilde{Y}_{F}^{\prime}\right)_{f r, W}=0$ and $\left(1 / \tilde{Y}_{F, I N}\right)\left(\tilde{Y}_{F}\right)_{f r, W}=$ 1.

Extensive computations have verified the proposed functional dependence: both Eqs. 32 and 33 are within $2.6 \%$ of the full numerical predictions, provided that $P$ is:

$P=1+0.158\left(\frac{T_{W}}{T_{I N}}\right)^{-1.12} L e^{0.32} D a_{s}^{0.75} \zeta^{0.13}$.

Typical streamwise profiles of the fuel wall gradient and fuel concentration are illustrated

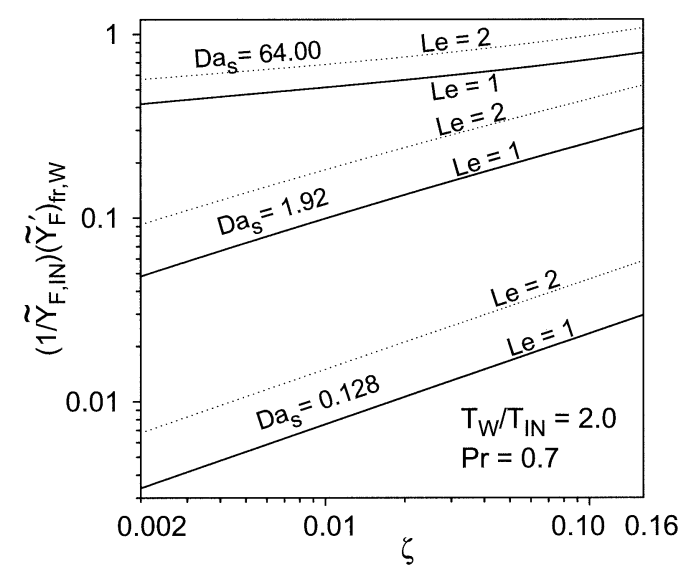

Fig. 3. Chemically frozen state: computed streamwise profiles of the non-dimensional transverse fuel wall gradient for three different surface Damköhler numbers, $D a_{s}$, and two Lewis numbers: $L e=1$ (solid lines) and $L e=2$ (dotted lines); $P r=0.7$ and $T_{W} / T_{I N}=2$.

in Figs. 3 and 4a, respectively. The accuracy of both Eqs. 32 and 33 over the entire allowable range of $\zeta, T_{W} / T_{I N}, L e, P r$, and $D a_{s}$ is illustrated in Fig. $4 \mathrm{~b}$.

\section{RESULTS AND DISCUSSION}

\section{Heterogeneous Ignition}

Considering half of the channel domain $(0 \leq$ $y \leq b)$, the local integrated heterogeneous fuel conversion rate per unit of channel width is,

$\dot{m}_{h, F}(x)=\int_{0}^{x}\left(\rho D \frac{\partial Y_{F}}{\partial y}\right)_{W} d x$.

The incoming mass fuel flow rate and the integrated fractional catalytic fuel conversion are,

$$
\dot{m}_{F, I N}=\rho_{I N} Y_{F, I N} U_{I N} b \quad \text { and }
$$

$I_{h, F}(x)=\dot{m}_{h, F}(x) / \dot{m}_{F, I N}$,

respectively. Using Eqs. 12, 14, 27, 35, 36, and the relation $\rho_{I N}^{2} D_{I N}=\rho_{W}^{2} D_{W}$, the fractional catalytic fuel conversion becomes:

$$
I_{h, F}(\zeta)=\frac{1}{L e} \int_{0}^{\zeta} \frac{1}{\sqrt{2 \operatorname{Pr} \zeta}}\left(1 / \tilde{Y}_{F, I N}\right)\left(\tilde{Y}_{F}^{\prime}\right)_{f r, W} d \zeta
$$



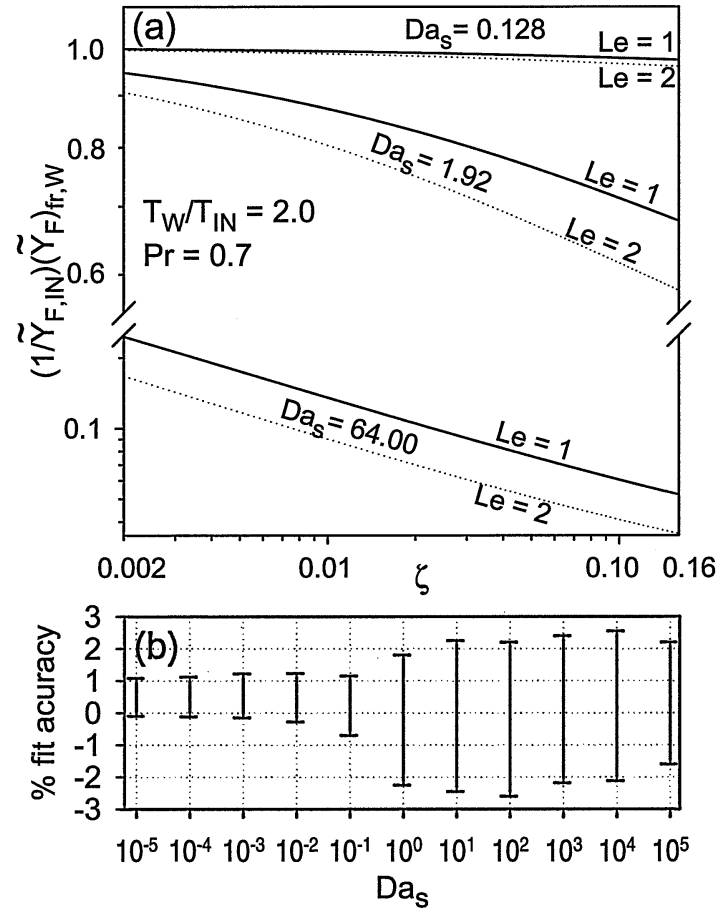

Fig. 4. Chemically frozen state: (a) computed streamwise profiles of the normalized fuel wall concentration for three different surface Damköhler numbers, $D a_{s}$, and two Lewis numbers: $L e=1$ (solid lines) and $L e=2$ (dotted lines); $\operatorname{Pr}=0.7$ and $T_{W} / T_{I N}=2.0$, (b) percentage accuracy (relative to the numerical predictions) of both Eqs. 32 and 33 versus the surface Damköhler number $D a_{s}$. The presented bars account for the entire parameter variation $0.002 \leq \zeta \leq 0.16,1.5 \leq T_{W} / T_{I N} \leq 3.0,0.9 \leq L e \leq 2.0$ and $0.5 \leq \operatorname{Pr} \leq 1.0$.

The catalytic conversion (Eq. 37) is directly linked to the fuel wall gradient. For small but non-zero values of $D a_{s}$, the fuel wall gradient behaves as $\approx G D a_{s}$ (see Eq. 32), and with the use of Eq. 30, $\left(1 / \tilde{Y}_{F, I N}\right)\left(\tilde{Y}_{F}^{\prime}\right)_{f r, W} \propto\left(T_{W} / T_{I N}\right)^{-1}$, that is, it is a decreasing function of $T_{W} / T_{I N}$. At large $D a_{s}$, however, $\left(1 / \tilde{Y}_{F, I N}\right)\left(\tilde{Y}_{F}^{\prime}\right)_{f r, W}$ behaves as $\left[\left(1 / \tilde{Y}_{F, I N}\right)\left(\tilde{Y}_{F}^{\prime}\right)_{f r, W}\right]_{D a_{s} \rightarrow \infty}$, which is a monotonically increasing function of $T_{W} / T_{I N}$ (see Eqs. 29 and 28). Figure 5 illustrates the aforementioned behavior. At sufficiently high $D a_{s}$ the surface reactions are fast compared to the fluid mechanical transport. An increase in $T_{W} /$ $T_{I N}$ through an increase of $T_{W}$ enhances the transport towards the wall (see Eq. 35 and consider that $\rho_{W} D_{W} \propto T_{W}$ in view of $\rho_{W}^{2} D_{W}=$ constant). The catalytic reactions can still cope with the increased surface loading, resulting in enhanced catalytic fuel conversion with increas-

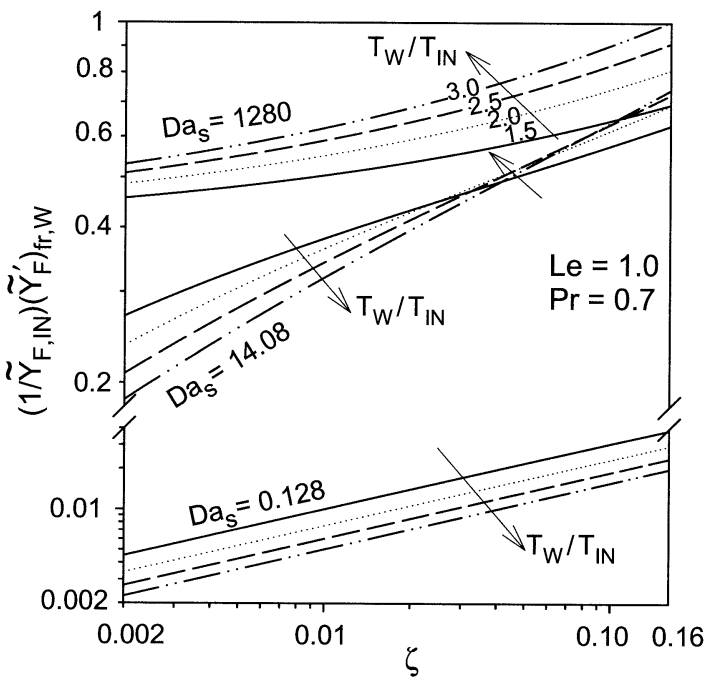

Fig. 5. Chemically frozen state: computed streamwise profiles of the non-dimensional transverse fuel wall gradient $\left(1 / \tilde{Y}_{F, I N}\right)\left(\tilde{Y}_{F}^{\prime}\right)_{f r, W}$ for various Damköhler numbers $D a_{s}$ and $T_{W} / T_{I N}: T_{W} / T_{I N}=1.5$ (solid lines), 2.0 (dotted lines), 2.5 (dashed lines), and 3.0 (dashed-doubled dotted lines); $L e=$ 1.0 and $\operatorname{Pr}=0.7$. The gradient $\left(1 / \tilde{Y}_{F, I N}\right)\left(\tilde{Y}_{F}^{\prime}\right)_{f r, W}$ is a decreasing function of $T_{W} / T_{I N}$ at low $D a_{s}$ (see $D a_{s}=$ $0.128)$ and an increasing function of $T_{W} / T_{I N}$ at high $D a_{s}$ $\left(D a_{s}=1280\right)$. The arrows indicate the direction of increasing $T_{W} / T_{I N}$.

ing $T_{W} / T_{I N}$. At low $D a_{s}$, on the other hand, the surface reactions cannot cope with the increased transport, resulting in a decrease of the catalytic fuel conversion with increasing $T_{W} /$ $T_{I N}$. The condition

$\partial\left[\left(1 / \tilde{Y}_{F, I N}\right)\left(\tilde{Y}_{F}^{\prime}\right)_{f r, W}\right] / \partial\left(T_{W} / T_{I N}\right)=0$

is, therefore, the appropriate definition of heterogeneous ignition. Equation 38 can be used to define a heterogeneous ignition Damköhler number, $D a_{s, i g}$. Figure 6 demonstrates the calculation of $D a_{s, i g}$ according to the definition of Eq. 38. $D a_{s, i g}$ depends strongly on $\zeta$ because finite-rate surface effects are more pronounced at shorter distances.

Of particular interest in practical applications is the attained heterogeneous fuel conversion under finite-rate surface chemistry operating conditions. The ratio of heterogeneous fuel conversion to the corresponding conversion at the mass-transport-limit, $\dot{m}_{h, F} /\left(\dot{m}_{h, F}\right)_{D a_{s} \rightarrow \infty}$, is presented in Fig. 7. This ratio is bounded between 0.55 and 0.68 over the entire allowable range of $\zeta, T_{W} / T_{I N}$ and $L e$. In catalysis, heter- 


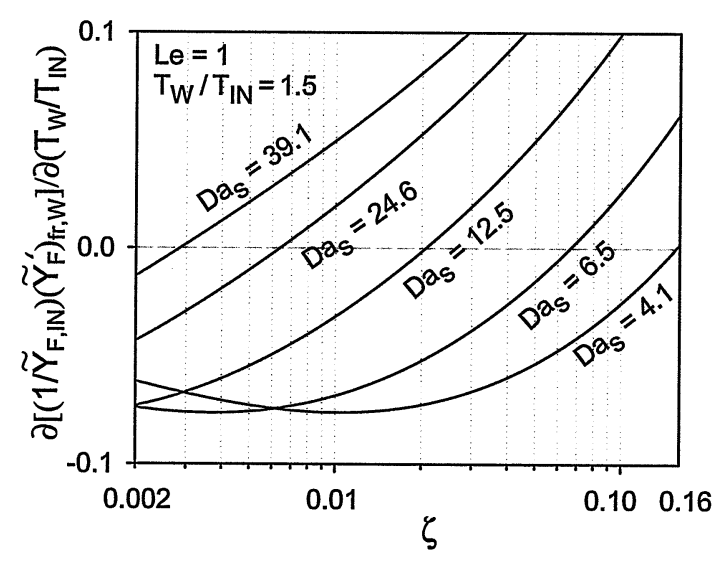

Fig. 6. Calculation of the heterogeneous ignition Damköhler number $\left(D a_{s, i g}\right)$ for $L e=1$ and $T_{W} / T_{I N}=1.5$. At heterogeneous ignition, the $\left(1 / \tilde{Y}_{F, I N}\right)\left(\tilde{Y}_{F}^{\prime}\right)_{f r, W}$ versus $T_{W} / T_{I N}$ profiles have a minimum according to Eq. 38. Da $a_{s, i g}$ has a strong dependence on the channel length $\zeta$.

ogeneous ignition is usually defined by the point of $50 \%$ relative fuel conversion (compared to the maximum attainable mass-transport-limited conversion), although definitions at relative conversions of $10 \%$ or $90 \%$ are also common $[30,31]$. In this respect, the precise value of the relative fuel conversion at $D a_{s, i g}$ is not of crucial. A simple functional relationship yielding (over the entire range of parameter variation) $D a_{s, i g}$ within $15 \%$ to those obtained from Eq. 38 is:

$$
D a_{s, i g}=\left(T_{W} / T_{I N}\right)^{3 / 2} L e^{-2 / 3} \zeta^{-1 / 2} .
$$

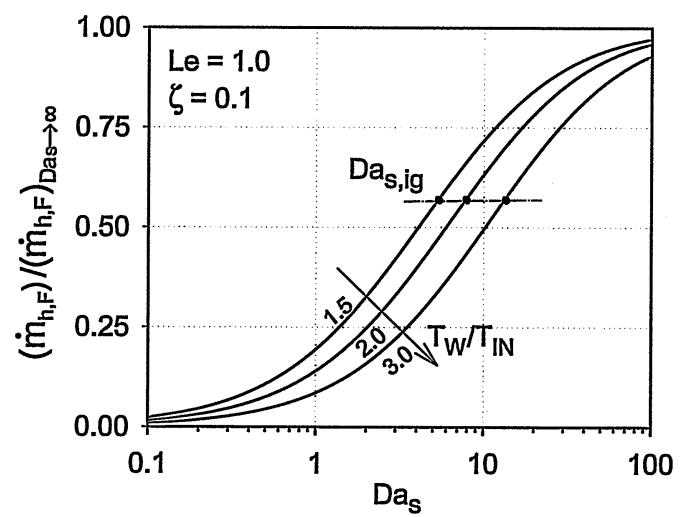

Fig. 7. Computed ratios of catalytic fuel conversion to the maximum attainable mass-transport-limited conversion versus $D a_{s}$, for $L e=1, \zeta=0.1$, and three $T_{W} / T_{I N}$. At heterogeneous ignition (calculated from Eq. 38 and shown by the solid points) the ratios $\dot{m}_{h, F} /\left(\dot{m}_{h, F}\right)_{D a_{s} \rightarrow \infty}$ are about 0.58 .
The heterogeneous ignition criterion of Eq. 39 contains all the dependencies on the characteristic flow, transport, chemical and geometrical parameters of the channel-flow configuration. The relation of Eq. 39 could be of interest to mid- or high-temperature CST catalysts that exhibit moderate catalyst temperature variations [32] because of their minimal upstream heat losses. Another application is the steam or $\mathrm{CO}_{2}$ reforming of $\mathrm{CH}_{4}$ to synthesis gas over catalysts [33]; the reforming is endothermic, requiring external heating that, in turn, imposes a nearly isothermal catalyst operation. Finally, nearly isothermal wall conditions can be encountered in laboratory-scale burners [14, 15].

\section{Homogeneous Ignition}

The asymptotic analysis of the weakly reactive state (details are given in Appendix A) leads to the homogeneous ignition distance, which is obtained from Eqs. A16 and A15. The first bracket of Eq. A16 involves the reactivity and exothermicity of the gaseous reaction; the inverse of this term is defined as:

$A=\frac{\left(T_{W}-T_{I N}\right)^{2+n_{F}}}{T_{W}^{2\left(1+n_{F}\right)}\left(R / E_{g}\right)^{1+n_{F}}\left(q / c_{p}\right)}$.

Introducing the functions

$F(\zeta)=\left(\frac{\left[\left(1 / \tilde{Y}_{F, I N}\right)\left(\tilde{Y}_{F}^{\prime}\right)_{f r, W}\right]_{D a_{s} \rightarrow \infty}}{\theta_{f r, W}^{\prime}}\right)^{n_{F}} \frac{1}{\left(\theta_{f r, W}^{\prime}\right)^{2}}$,

and

$H\left(\zeta ; D a_{s}\right)=\frac{\left[\left(1 / \tilde{Y}_{F, I N}\right) /\left(\tilde{Y}_{F}^{\prime}\right)\right]_{D a_{s} \rightarrow \infty}}{G P D a_{s}}$,

and further using Eq. 32, the second bracket of Eq. A16 becomes:

$$
\begin{gathered}
\left(\frac{\left(1 / \tilde{Y}_{F, I N}\right)\left(\tilde{Y}_{F}^{\prime}\right)_{f r, W}}{\theta_{f r, W}^{\prime}}\right)^{n_{F}} \frac{1}{\left(\theta_{f r, W}^{\prime}\right)^{2}}=F(\zeta) \\
\cdot\left[\frac{1}{1+H\left(\zeta ; D a_{s}\right)}\right]^{n_{F}} \cdot
\end{gathered}
$$

The third bracket of Eq. A16 is the inverse of a characteristic gaseous reaction time 


$$
\begin{aligned}
\tau_{g, c h}= & {\left[B_{g}(p \bar{W} / R)^{n_{F}-1} W_{F}^{1-n_{F}} T_{W}^{1+\gamma-n_{F}} Y_{F, I N}^{n_{F}}\right.} \\
& \left.\cdot \exp \left(-E_{g} / R T_{W}\right)\right]^{-1},
\end{aligned}
$$

and, finally, the last bracket of Eq. A16 after substitution of Eq. 13 becomes a characteristic transverse diffusion time scale:

$\tau_{d}=\left[\operatorname{RePrb} / U_{I N}\right]=b^{2} / \alpha_{t h, I N}$.

Defining a gas-phase Damköhler number as

$D a_{g}=\tau_{d} / \tau_{g, c h}$,

substituting Eqs. 41 through 43 in Eq. A16, and solving for the ignition distance $\zeta_{i g}\left(=s_{i g} / P r\right)$ :

$$
\begin{aligned}
\zeta_{i g} F\left(\zeta_{i g}\right)= & \frac{1}{2 P r} A \frac{1}{D a_{g}}\left[1+H\left(\zeta_{i g} ; D a_{s}\right)\right]^{n_{F}} \\
& \cdot \Delta_{c r}^{*}\left(\zeta_{i g} ; D a_{s}\right),
\end{aligned}
$$

with $\Delta_{c r}^{*}$ the critical ignition Damköhler number, obtained from the solution of Eq. A15. The homogeneous ignition criterion of Eq. 47 is valid over the range $0.002 \leq \zeta \leq 0.16,1.5 \leq$ $T_{W} / T_{I N} \leq 3.0,0.9 \leq L e \leq 2.0$, and $0.5 \leq$ $\operatorname{Pr} \leq 1.0$.

The main $\zeta$-dependence in Eq. 47 comes from the $\zeta F(\zeta)$ term because of its dominant $\zeta$ contribution; moreover, $\zeta F(\zeta)$ is a monotonically increasing function of $\zeta$. For the same $T_{W}, T_{I N}$, $c_{p}$, and $q$ (e.g., fixed value of $A$ ), the right side of Eq. 47 is controlled by the gaseous $\left(D a_{g}\right)$ and surface $\left(D a_{s}\right)$ reactivities. The quantity $[1+$ $\left.H\left(\zeta_{i g} ; D a_{s}\right)\right]^{n_{F}} \Delta_{c r}^{*}\left(\zeta_{i g} ; D a_{s}\right)$ is, as further discussed in Appendix B, smaller than unity and decreases monotonically with decreasing $D a_{s}$. In conjunction with the aforementioned $\zeta F(\zeta)$ dependence, Eq. 47 implies that homogeneous ignition is favored for either small $D a_{s}$ or large $D a_{g}$. The increased gas-phase reactivity promotes homogeneous ignition directly whereas the reduced surface reactivity promotes ignition indirectly through a larger near-wall fuel excess. In the limit $D a_{s} \rightarrow \infty, H\left(\zeta ; D a_{s}\right) \rightarrow 0$ (see Eq. 42 ), so that Eq. 47 reduces to the homogeneous ignition criterion $\zeta_{i g} F\left(\zeta_{i g}\right)=$ $\left[\Delta_{c r}^{*}\left(\zeta_{i g}\right)\right]_{D a_{s} \rightarrow \infty}(A / 2 P r)\left(1 / D a_{g}\right)$ of infinitely fast surface chemistry [16]. For direct comparisons with the mass-transport-limited solution, it is instructive to define an effective transverse diffusion time scale

$$
\begin{aligned}
\tau_{e f f, d}= & \tau_{d} /\left[\left(1+H\left(\zeta ; D a_{s}\right)\right)^{n_{F}} \Delta_{c r}^{*}\left(\zeta ; D a_{s}\right) /\right. \\
& \left.\cdot\left[\Delta_{c r}^{*}(\zeta)\right]_{D a_{s} \rightarrow \infty}\right],
\end{aligned}
$$

such that the homogeneous ignition criterion of Eq. 47 can be written equivalently as:

$$
\zeta_{i g} F\left(\zeta_{i g}\right)=\left[\Delta_{c r}^{*}\left(\zeta_{i g}\right)\right]_{D a_{s} \rightarrow \infty}(A / 2 P r)\left(\tau_{c h} / \tau_{e f f, d}\right) .
$$

Under mass-transport-limited conditions, the effective transverse transport time scale $\tau_{e f f, d}$ is simply the diffusion time scale $\tau_{d}$. Finite rate surface kinetics, on the other hand, reduce the transverse transport rates by increasing the effective transverse diffusion time scale (as discussed in Appendix B, the bracketed quantity of Eq. 48 is less or equal to unity and decreases monotonically with decreasing $D a_{s}$ ). To investigate homogeneous ignition, Eq. A15 must solved numerically to provide $\Delta_{c r}^{*}$; details are provided in Appendix B for a gaseous reaction that is first-order with respect to the fuel $\left(n_{F}=\right.$ $1)$.

\section{Sensitivity of Homogeneous Ignition on Surface and Gaseous Reaction Pathways}

To assess the importance of each reaction pathway on homogeneous ignition, an application of Eq. 47 is presented next. A propane-oxygennitrogen mixture is considered, with incoming composition $Y_{C 3 H 8, I N}=0.0332, Y_{O 2, I N}=$ $0.3764(\phi=0.32)$, and balance nitrogen. The other parameters are $T_{I N}=600 \mathrm{~K}, p=1$ bar, $W_{F}=44 \mathrm{~kg} / \mathrm{kmol}, W_{O}=32 \mathrm{~kg} / \mathrm{kmol}, \bar{W}=29$ $\mathrm{kg} / \mathrm{kmol}, \nu_{O}=5, q=46400 \mathrm{~kJ} / \mathrm{kgC}_{3} \mathrm{H}_{8}, c_{p}=$ $1.29 \mathrm{~kJ} / \mathrm{kgK}, P r=0.7$, and $L e=1.85$. A gaseous reaction first-order with respect to propane and zero-order with respect to oxygen is considered, $\left(\dot{\omega}_{F} / W_{F}\right)=B_{g}\left[C_{C 3 H 8}\right] \exp \left(-E_{g} /\right.$ $R T)$. The activation energy is $E_{g}=1.675 \times$ $10^{5} \mathrm{~kJ} / \mathrm{kmol}$ [34] and the pre-exponential constant is $B_{g}=3.42 \times 10^{10} \mathrm{sec}^{-1}$ so that the resulting reaction rate matches the global reaction rate of Westbrook and Dryer [35] proposed for propane flame applications at about $1300 \mathrm{~K}$. It is noted that the above gaseous reaction parameters are intended for the application of Eq. 47 rather than for precise homogeneous ignition calculations in practical systems. Apart 


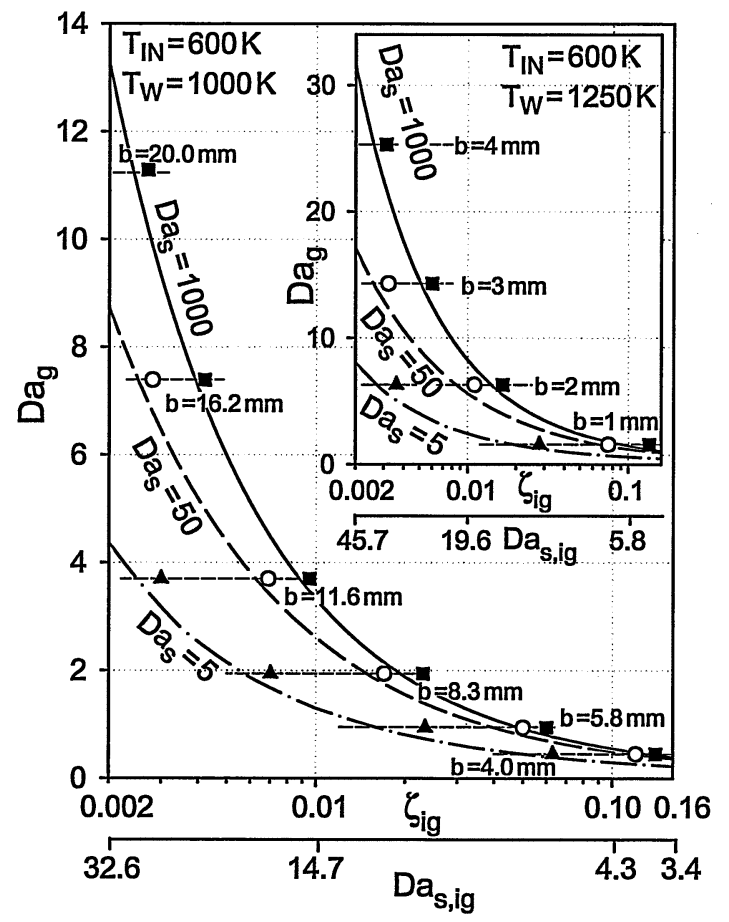

Fig. 8. Homogeneous ignition distances $\zeta_{i g}$ versus gaseous Damköhler numbers, $D a_{g}$, for a propane-oxygen-nitrogen mixture with $T_{I N}=600 \mathrm{~K}, T_{W}=1000 \mathrm{~K}, p=1 \mathrm{bar}, L e=$ 1.85, and three different surface Damköhler numbers; the insert figure corresponds to $T_{W}=1250 \mathrm{~K}$. The lines are analytical predictions from Eq. 47 (solid lines $D a_{s}=10^{3}$, dashed lines $D a_{s}=50$, and dashed-dotted lines $D a_{s}=5$ ) and the symbols are numerical simulations (solid squares $D a_{s}=10^{3}$, open circles $D a_{s}=50$, and solid triangles $\left.D a_{s}=5\right)$. The horizontal axes provide also the corresponding heterogeneous ignition Damköhler numbers $D a_{s, i g}$ calculated from the ignition criterion of Eq. 38.

from the already known limitations of global steps, accurate homogeneous ignition CST predictions require additional consideration of the hetero/homogeneous coupling of intermediate radical species [14, 15]; in that, a global gaseous reaction should have higher apparent activation energy (compared to that of the purely homogeneous case) to compensate for the inhibition induced by radical adsorption reactions [17].

Plots of non-dimensional ignition distances versus $D a_{g}$ are presented in Fig. 8 for $T_{W}=$ $1000 \mathrm{~K}$ and three different $D a_{s} ; D a_{s}=10^{3}$ is close to the mass-transport-limited solution. The insert of Fig. 8 illustrates the corresponding plots for $T_{W}=1250 \mathrm{~K}$. The lines in Fig. 8 are analytical predictions from Eq. 47 and the symbols are numerical simulations. The definition
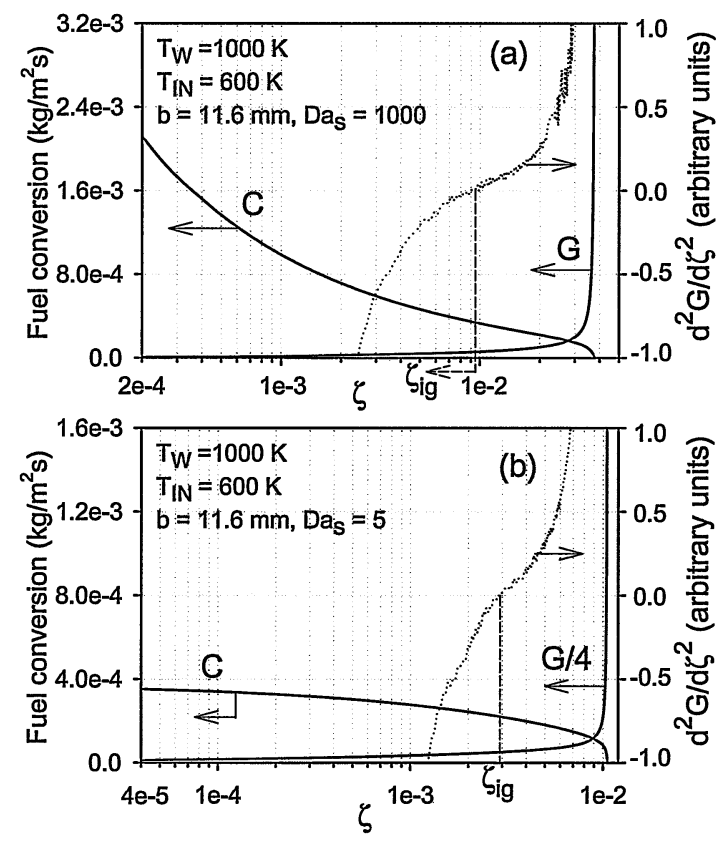

Fig. 9. Computed streamwise profiles of the local catalytic $(C)$ and gaseous $(G)$ fuel conversion rates for $T_{I N}=600 \mathrm{~K}$, $T_{W}=1000 \mathrm{~K}, b=11.6 \mathrm{~mm}$, and two different surface Damköhler numbers: (a) $D a_{s}=10^{3}$ and (b) $D a_{s}=5$. For clarity, in (b) the $G$ profile is divided by a factor of four. The onset of homogeneous ignition is defined by the inflection point of the $G$ profile.

of homogeneous ignition in the numerical simulations is illustrated in Fig. 9 for $T_{I N}=600 \mathrm{~K}$, $T_{W}=1000 \mathrm{~K}, b=11.6 \mathrm{~mm}$, and two surface reactivities. The local catalytic $(C)$ and gaseous $(G)$ conversions are plotted versus $\zeta$; for direct comparison with the catalytic surface rate, the volumetric gaseous rate has been integrated over the channel half-height. The gaseous conversion rate $(G)$ increases initially very slowly and after a certain streamwise distance that is shorter for the lower $D a_{s}$ case, it rises exponentially. The inflection point of the $G$ profile $\left(d^{2} G / d \zeta^{2}=0\right)$ is used in Fig. 9 to define the onset of homogeneous ignition. The presence of such inflection points can be explained by the competition between fuel depletion and mixture heatup. Initially, $d G / d \zeta$ drops as the near-wall fuel depletion increases with streamwise distance (see Fig. 2a) and at the same time the mixture heating is not yet significant. However, as the thermal effects become important, the Arrhenius exponential dominates and $d G / d \zeta$ increases again. This definition yields ignition 


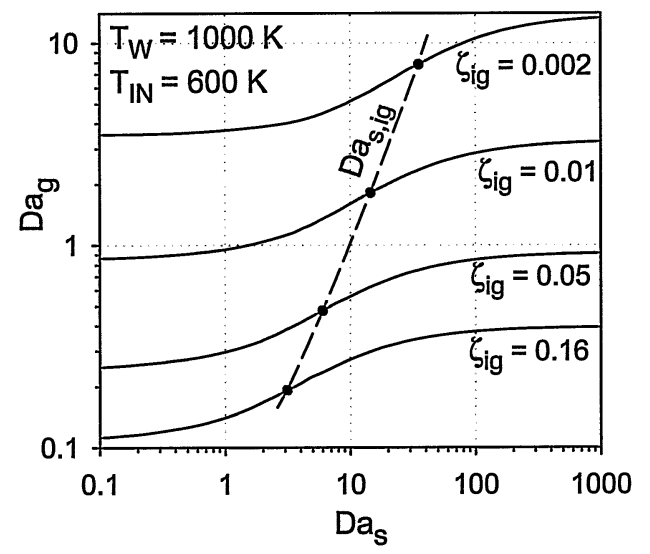

Fig. 10. Lines of constant homogeneous ignition distances $\left(\zeta_{i g}\right)$ for different combinations of surface and gaseous Damköhler numbers; $T_{I N}=600 \mathrm{~K}, T_{W}=1000 \mathrm{~K}$, the other parameters being as in Fig. 8. The solid points are the heterogeneous ignition Damköhler numbers, $D a_{s, i g}$, calculated from the ignition criterion of Eq. 38.

distances at the base of the rising $G$-profile, a region where the basic approximations of the mathematical formulation hold, since at the location of the exponential $G$ rise the asymptotic analysis breaks down. In addition, this definition provides ignition distances in good agreement to those predicted analytically. A definition of homogeneous ignition exactly at the point of the exponential $G$ rise would give $\zeta_{i g}$ a factor of three larger than those of the analytical predictions; such factors are inherent to all first-order asymptotic analyses for the reasons elaborated in Law and Law [36].

The comparisons between analytical and numerical predictions of Fig. 8 are very favorable. The analytical predictions reproduce not only the trends but also the quantitative features of the numerical predictions. As evidenced from Fig. 8, reduction of $D a_{s}$ leads to shorter ignition distances $\zeta_{i g}$. Alternately, for a fixed $D a_{s}$, reduction of the channel half-height $b$ results in an increase of the ignition distance $\zeta_{i g}$; this is because the surface-to-volume ratio increases with decreasing $b$ and, hence, catalytic conversion is favored against homogeneous conversion. The sensitivity of the ignition distance on both reaction pathways is discussed with the aid of Fig. 10 (referring to the same conditions as Fig. 8), where lines of constant $\zeta_{i g}$ are presented for various $D a_{g}$ and $D a_{s}$. As seen from the criterion of Eq. 47 or 49, there are infinite combinations of $D a_{g}$ and $D a_{s}$ (such that the terms $D a_{g}^{-1}\left[1+H\left(\zeta_{i g} ; D a_{s}\right)\right]^{n_{F}} \Delta_{c r}^{*}\left(\zeta_{i g} ; D a_{s}\right)$ or $\tau_{c h} / \tau_{\text {eff,d }}$ are constant) that produce the same $\zeta_{i g}$. When a slow surface reaction (e.g., more pronounced near-wall fuel excess) is coupled to a slow gaseous reaction, it can produce the same $\zeta_{i g}$ (although not necessarily the same postignition flame structure) with that of a faster surface reaction (reduced near-wall fuel excess) coupled to a faster gaseous reaction. The existence of multiple combinations of gaseous and surface reactivities producing the same $\zeta_{i g}$ complicates the validation of hetero/homogeneous reaction schemes with homogeneous ignition studies; to circumvent such complications, experimental input is required either on the catalytic processes over the pre-ignition zone or on the flame structure over the post-ignition zone. As seen from Fig. 10, the sensitivity of the homogeneous ignition distance to the surface pathway is more pronounced around $D a_{s}=$ $D a_{s, i g}$. Even then, $\zeta_{i g}$ is most sensitive to the gaseous rather than to the surface pathway; for example, the $10 \%$ to $90 \%$ rise in $D a_{g}$ is accomplished by a factor of about 2.5 increase in $D a_{g}$ (from 0.14 to 0.36 when $\zeta_{i g}=0.16$ and from 4.5 to 12.3 when $\zeta_{i g}=0.002$ ); the corresponding increase in $D a_{s}$ is much larger (from 0.9-68; e.g., a factor of 75 when $\zeta_{i g}=0.16$ and from 6 to 327 e.g. a factor of 55 when $\zeta_{i g}=0.002$ ). The implications to homogeneous ignition simulations of practical systems are that an uncertainty in the gaseous reaction scheme carries considerably more weight than a corresponding uncertainty in the surface reaction scheme. This is consistent to our recent CST studies of $\mathrm{H}_{2}$ /air mixtures [15], where various elementary hetero/ homogeneous reaction schemes were evaluated against measured homogeneous ignition characteristics; there were large differences among the predictions of the various hetero/homogeneous schemes and these differences were ascribed primarily to the gaseous reaction pathway.

\section{CONCLUSIONS}

The heterogeneous and homogeneous ignition of fuel-lean premixtures was investigated analytically and numerically in plane channel-flow 
configurations with catalytically-active isothermal walls. The following are the key conclusions of this study.

1) A closed-form heterogeneous ignition criterion was derived for a first-order with respect to the fuel global catalytic reaction. The criterion is $D a_{s, i g}=\left(T_{W} / T_{I N}\right)^{3 / 2} \zeta^{-1 / 2} L e^{-2 / 3}$, with $D a_{s, i g}$ a corresponding heterogeneous ignition Damköhler number, valid over the range $0.002 \leq \zeta \leq 0.16,1.5 \leq T_{W} / T_{I N} \leq$ 3.0, $0.9 \leq L e \leq 2.0$, and $0.5 \leq P r \leq 1.0$.

2) A closed-form homogeneous ignition criterion was established (Eq. 47), which included explicitly the effect of gaseous and surface reactivities, that is, the relevant Damköhler numbers $D a_{g}$ and $D a_{s}$. The range of applicability of the homogeneous ignition criterion is the same as that of the heterogeneous criterion presented above. Finite-rate rate surface kinetics (decreasing $D a_{s}$ ) promote homogeneous ignition due to the associated increase of the near-wall fuel levels. The effect of finite-rate surface kinetics is manifested in the homogeneous ignition criterion via an increase of the characteristic transverse diffusion time scale with decreasing $D a_{s}$.

3) Numerical simulations with fuel-lean propane-oxygen-nitrogen mixtures have shown that, over a wide range of parameter variation, the analytical homogeneous ignition distances are in good agreement with those numerically predicted.

4) The homogeneous ignition distance is much more sensitive to the gaseous rather than to the surface pathway, the reason being that the latter affects homogeneous ignition indirectly via the transport of the deficient reactant (fuel). In addition, there exist infinite combinations of surface and gaseous reactivities ( $D a_{s}$ and $D a_{g}$, respectively) yielding the same homogeneous ignition distance, hence suggesting caution in the interpretation of CST experiments.

Support was provided by the Swiss Federal Office of Energy (BFE), Federal Office of Education and Technology (BBT) under contract No. KTI 5344.2 EBS, and Alstom Power Technology of Switzerland.

\section{REFERENCES}

1. Beebe, K. W., Cairns, K. D., Pareek, V. K., Nickolas, S. G., Schlatter, J. C., and Tsuchiya, T., Catalysis Today 59:95-115 (2000).

2. Forzatti, P., Appl. Catalysis A: General 222:221-236 (2001).

3. Farrauto, R. J., Hobson, M. C., Kennelly, T., and Waterman, E. M., Appl. Catalysis A: General 81:227237 (1992).

4. Pfefferle, W. C., and Pfefferle, L. D., Prog. Energy Combust. Sci. 12:25-41 (1985).

5. Machida, M., Eguchi, K., and Arai, H., J. Catalysis 120:377-386 (1989).

6. Dalla Betta, R., Ribeiro, F. H., Shoji, T., Tsurumi, K., Ezawa, N., and Nickolas, S. G., U.S. Patent No. 5250489, 1993.

7. McCarty, J., Wong, V. L., and Wood, B. J., U.S. Patent No. 6015285, 2000.

8. Hickman, D. A., and Schmidt, L. D., AIChE 39(7): 1164-1177 (1993).

9. Deutschmann, O., Schmidt, R., Behrendt, F., and Warnatz, J., Proc. Combust. Institute 26:1747-1754 (1996).

10. Hellsing, B., Kasemo, B., and Zhdanov, V. P., J. Catalysis 132:210-228 (1991).

11. Aghalayam, P., Park, Y. K., and Vlachos, D. G., Proc. Combust. Institute 28:1331-1339 (2000).

12. Dogwiler, U., Benz, P., and Mantzaras, J., Combust. Flame 116:243-258 (1999).

13. Deutschmann, O., and Schmidt, L. D., AIChE 44(11): 2465-2477 (1998).

14. Dogwiler, U., Mantzaras, J., Benz, P., Kaeppeli, B., Bombach, R., and Arnold, A., Proc. Combust. Institute 27:2275-2282 (1998).

15. Appel, C., Mantzaras, J., Schaeren, R., et al., Combust. Flame 128:340-368 (2002).

16. Mantzaras, J., and Benz, P., Combust. Flame 119:455472 (1999).

17. Mantzaras, J., Appel, C., and Benz, P., Proc. Combust. Institute 28:1349-1357 (2000).

18. Glassman, I., Combustion, 3rd Edition. Academic Press, London, 1996, p. 156.

19. Law, C. K., and Sivashinsky, G. S., Combust. Sci. Technol. 29:277-286 (1982).

20. Law, C. K., and Chung, S. H., Combust. Sci. Technol. 32:307-312 (1983).

21. Trevino, C., Combust. Sci. Technol. 30:213-229 (1983).

22. Trevino, C., and Fernandez-Pello, A. C., Combust. Sci. Technol. 26:245-251 (1981).

23. Trevino, C., and Peters, N., Combust. Flame 61:39-49 (1985).

24. Hiam, L., Wise, H., and Chaikin, S., J. Catalysis 9:272-276 (1968).

25. Trimm, D. L., and Lam, C.-W., Chem. Engineering Sci. 35:1405-1413 (1980).

26. Markatou, P., Pfefferle, L. D., and Smooke, M. D., Combust. Flame 93:185-201 (1993).

27. Williams, F. A., Combustion Theory, 2nd Edition. Benjamin/Cummings, CA, 1985, p. 486.

28. Raja, L. L., Kee, R. J., Deutschmann, O., Warnatz, J., and Schmidt, L. D., Catalysis Today 59:47-60 (2000). 
29. Shah, R. K., and London, A. L., Laminar Flow Forced Convection in Ducts. Academic Press, New York, 1978, p. 50 .

30. Arnone, S., Busca, G., Lisi, L., Milella, F., Russo, G., and Turco, M., Proc. Combust. Institute 27:2293-2299 (1998).

31. Artizzu-Duart, P., Millet, J. M., Guilhaume, N., Garbowski, E., and Primet, M., Catalysis Today 59:163-177 (2000).

32. Dalla Betta, R. A., and Rostrup-Nielsen, T., Catalysis Today 47:369-375 (1999).

33. Choudhary, V. R., Uphade, B. S., and Mamman, A. S., Appl. Catalysis A: General 168:33-46 (1998).

34. Puri, I. K., and Seshadri, K., Combust. Flame 65:137150 (1986).

35. Westbrook, C. K., and Dryer, F. L., Combust. Sci. Technol. 27:31-43 (1981).

36. Law, C. K., and Law, H. K., Combust. Sci. Technol. 25:1-8 (1981).

Received 9 October 2001; revised 2 April 2002; accepted 19 April 2002

\section{APPENDIX A}

\section{Asymptotic Analysis of the Weakly Reactive \\ State}

The gaseous reactive state is formulated by Eqs. 15 through 18 with the initial and boundary conditions of Eqs. 19, 20, 23, and 24. In terms of the non-dimensional variables

$$
\begin{aligned}
\tilde{T} & =\frac{c_{p} T}{q}, \quad \beta=\frac{\tilde{T}_{W}-\tilde{T}_{I N}}{\tilde{T}_{W}}, \\
\tilde{T}_{a} & =\frac{E_{g}}{R T_{W}}, \quad n=n_{O}+n_{F}, \\
\tilde{\omega} & =(1-\beta \theta)^{1+\gamma-n^{-}} \tilde{Y}_{F}^{n_{F}} \tilde{Y}_{O}^{n_{O}} \exp \left(\frac{-\tilde{T}_{a}}{1-\beta \theta}\right),
\end{aligned}
$$

$\tilde{B}=B_{g}\left(\frac{R e b}{U_{I N}}\right)\left(\frac{p \bar{W}}{R W_{F}}\right)^{n-1} \nu_{O}^{n_{O}}\left(q / c_{p}\right)^{1+\gamma-n} \tilde{T}_{W}^{1+\gamma-n}$,

and the normalized coordinate $\xi$,

$$
\xi=\frac{Z(\eta)}{Z\left(\eta^{*}\right)} \equiv \frac{\int_{0}^{\eta} \exp \left(-\operatorname{Pr} \int_{0}^{\eta} f d \eta\right) d \eta}{\int_{0}^{\eta^{*}} \exp \left(-\operatorname{Pr} \int_{0}^{\eta} f d \eta\right) d \eta},
$$

the inner-zone expansion for the temperature, $\theta_{i n r}=\theta_{f r}-\epsilon \varphi$, results via an asymptotic matching process (in the same manner as in our earlier mass-transport-limited work [16]) to the following differential equation for the perturbation quantity $\varphi$ :

$\frac{d^{2} \varphi}{d \chi^{2}}=-2 s \frac{\epsilon \operatorname{Pr} \tilde{B} \tilde{\omega}}{A_{1}^{2} \beta \tilde{T}_{W}\left(\theta_{f r, W}^{\prime}\right)^{2}}$

with boundary conditions $\varphi(0)=0$ and $\partial \varphi / \partial \chi=$ 0 at $\chi \rightarrow \infty$, where

$\chi=\theta_{f r, W}^{\prime} \xi Z\left(\eta^{*}\right) / \epsilon \quad$ and

$\epsilon=\frac{1}{\beta \tilde{T}_{a}}=\frac{T_{W}^{2} R}{\left(T_{W}-T_{I N}\right) E_{g}}$.

The asymptotic analysis for the inner-zone of the fuel is discussed next, as it differs from the previous mass-transport analysis [16]. The inner-zone fuel expansion is expressed as:

$\tilde{Y}_{F, i n r}=\tilde{Y}_{F, f r}-\epsilon \psi$

with $\psi$ the fuel perturbation. The chemically frozen solution near the wall is

$$
\begin{aligned}
\left(1 / \tilde{Y}_{F, I N}\right) \tilde{Y}_{F, f r}= & \left(1 / \tilde{Y}_{F, I N}\right)\left(\tilde{Y}_{F}\right)_{f r, W}+\left(1 / \tilde{Y}_{F, I N}\right) \\
& \cdot\left(\tilde{Y}_{F}^{\prime}\right)_{f r, W} \eta+O\left(\eta^{2}\right) .
\end{aligned}
$$

The temperature and fuel perturbations $\varphi$ and $\psi$ can be linked as,

$\psi=\varphi \alpha \frac{\left(\tilde{Y}_{F}^{\prime}\right)_{f r, W}}{\theta_{f r, W}^{\prime}}$,

where $\alpha$ is to be defined next.

With the aid of Eqs. A6 and A9, the innerzone expansion of Eq. A7 becomes

$$
\begin{aligned}
\left(1 / \tilde{Y}_{F, I N}\right) \tilde{Y}_{F, i n r}= & \epsilon \frac{\left(1 / \tilde{Y}_{F, I N}\right)\left(\tilde{Y}_{F}^{\prime}\right)_{f r, W}}{\theta_{f r, W}^{\prime}} \\
& \cdot(Q+\chi-\alpha \varphi) .
\end{aligned}
$$

with

$$
Q=\frac{\left(1 / \tilde{Y}_{F, I N}\right)\left(\tilde{Y}_{F}\right)_{f r, W}}{\epsilon\left[\left(1 / \tilde{Y}_{F, I N}\right)\left(\tilde{Y}_{F}^{\prime}\right)_{f r, W} / \theta_{f r, W}^{\prime}\right]}=\frac{\theta_{f r, W}^{\prime}}{\epsilon G D a_{s}},
$$

Using Eq. A4, the fuel conservation equation (Eq. 17) becomes 


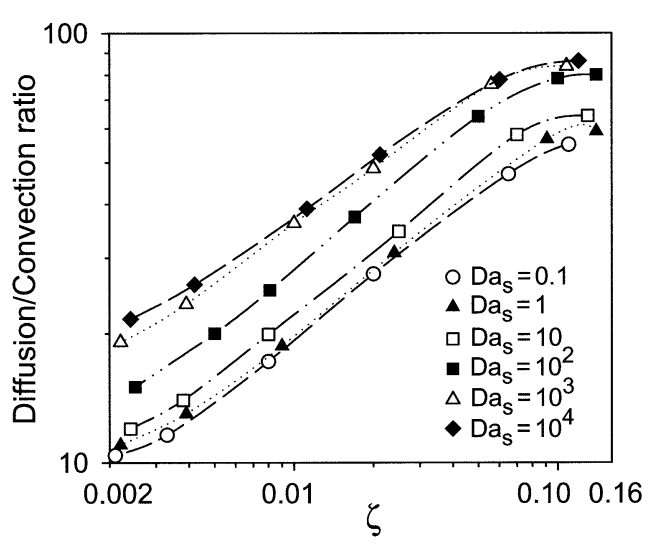

Fig. 11. Numerically predicted ratios of diffusion to streamwise convection (see Eq. A12), at the streamwise position of homogeneous ignition and the transverse location $y / b=$ $0.005 ; T_{W} / T_{I N}=1.67$, Le $=1.85, \operatorname{Pr}=0.7$. The streamwise convection becomes more important at lower $D a_{s}$ or shorter streamwise distances $\zeta$.

$$
\begin{aligned}
S c^{-1} \frac{\partial^{2} \tilde{Y}_{F}}{\partial \xi^{2}}= & 2 s\left[\left(\frac{A_{2}}{A_{1}}\right)^{2}\left(\frac{\partial f}{\partial \eta}\right) \frac{\partial \tilde{Y}_{F}}{\partial s}-\left(\frac{A_{2}}{A_{1}}\right)\right. \\
& \left.\cdot\left(\frac{\partial f}{\partial s}\right) \frac{\partial \tilde{Y}_{F}}{\partial \xi}\right]+2 s\left(\frac{A_{2}}{A_{1}}\right)^{2} \frac{\tilde{B} \tilde{\omega}}{\beta \tilde{T}_{W}},
\end{aligned}
$$

with boundary conditions $\tilde{Y}_{F}=\left(\tilde{Y}_{F}\right)_{f r, W}$ at $\xi=$ 0 and $\partial \tilde{Y}_{F} / \partial \xi=0$ at $\xi=1$. The functions $A_{1}$ and $A_{2}$ are $A_{1}=d Z / d \eta, A_{2}=Z\left(\eta^{*}\right)$. Equation A10 is further substituted in A12 and the leading term balancing the reaction terms is sought. The first convective term on the right side of Eq. A12 deserves attention, notwithstanding its apparent $O\left(\epsilon^{3}\right)$ magnitude, because of the included factor $\partial \tilde{Y}_{F} / \partial s$. Finite rate surface chemistry can induce large streamwise gradients $\partial \tilde{Y}_{F} / \partial s$, particularly at short $s$ (or $\zeta$ ) as shown in Fig. 4. This effect is seen in Fig. 11 where numerically computed diffusion-to-convection ratios, $-\left[S c^{-1} \partial^{2} \tilde{Y}_{F} / \partial \xi^{2}\right] /\left[2 s\left(A_{2} /\right.\right.$ $\left.\left.A_{1}\right)^{2}(\partial f / \partial \eta)\left(\partial \tilde{Y}_{F} / \partial s\right)\right]$, are presented for various $D a_{s}$ at the streamwise locations of homogeneous ignition and the transverse position $y / b=0.005$. The gaseous reaction parameters in the computations of Fig. 11 correspond to $\epsilon=0.12$. A diffusion-to-convection ratio of at least ten is guaranteed even for $D a_{s}=0.1$. Consequently, Eq. A12 with the additional use of Eq. A9 reduces to: $\frac{d^{2} \varphi}{d \chi^{2}}=-2 s \frac{\epsilon S c \tilde{B} \tilde{\omega}}{A_{1}^{2} \theta_{f r, W}^{\prime}\left(\tilde{Y}_{F}^{\prime}\right)_{f r, W} \alpha}$.

The boundary conditions of Eq. A13 are the same with those of Eq. A5. Comparison of Eqs. A13 and A5 shows that $\alpha=L e \beta \tilde{T}_{W} \theta_{f r, W}^{\prime}$ $\left(\tilde{Y}_{F}^{\prime}\right)_{f r, W}$ and substituting $\beta$ from Eq. A1,

$$
\alpha=\frac{\operatorname{Le}\left(\tilde{T}_{W}-\tilde{T}_{I N}\right)}{\tilde{Y}_{F, I N}}\left[\frac{\theta_{f r, W}^{\prime}}{\left(1 / \tilde{Y}_{F, I N}\right)\left(\tilde{Y}_{F}^{\prime}\right)_{f r, W}}\right] .
$$

Considering - without loss of generality-a gaseous reaction rate that is zero-order with respect to the oxidizer $\left(n_{O}=0\right)$, and substituting Eqs. A2, A6, and A10 into Eq. A13:

$\frac{d^{2} \varphi}{d \chi^{2}}=-\Delta_{c r}(Q+\chi-\alpha \varphi)^{n_{F}} \exp (\varphi-\chi)$,

with boundary conditions $\varphi(0)=0$ and $\partial \varphi / \partial \chi=$ 0 at $\chi \rightarrow \infty . \Delta_{c r}$ is the critical ignition Damköhler number, which, under the aforementioned $n_{O}=0$ dependence of the gaseous reaction rate, is

$$
\begin{aligned}
\Delta_{c r}= & 2 s\left[\frac{T_{W}^{2\left(1+n_{F}\right)}\left(R / E_{g}\right)^{1+n_{F}}\left(q / c_{p}\right)}{\left(T_{W}-T_{I N}\right)^{2+n_{F}}}\right] \\
& \cdot\left[\left(\frac{\left(1 / \tilde{Y}_{F, I N}\right)\left(\tilde{Y}_{F}^{\prime}\right)_{f r, W}}{\theta_{f r, W}^{\prime}}\right)^{n_{F}} \frac{1}{\left(\theta_{f r, W}^{\prime}\right)^{2}}\right] \\
& \cdot\left[B_{g}(p \bar{W} / R)^{n_{F}-1} W_{F}^{1-n_{F}} T_{W}^{1+\gamma-n_{F}} Y_{F, I N}^{n_{F}}\right. \\
& \left.\cdot \exp \left(-E_{g} / R T_{W}\right)\right]\left[\operatorname{RePrb} / U_{I N}\right] .
\end{aligned}
$$

\section{APPENDIX B}

\section{Critical Ignition Damköhler Numbers}

The critical ignition Damköhler number $\Delta_{c r}^{*}$ is determined by the turning point in the wallgradient perturbation $\varphi_{\chi}(0)$ versus $\Delta_{c r}$ profile; no steady solutions are possible for $\Delta_{c r}>\Delta_{c r}^{*}$, indicating that the system has undergone thermal runaway. For fixed values of $\alpha$ and $Q$, numerical integration of Eq. A15 is carried out and the particular value of $\Delta_{c r}$ is sought for which a unique solution for the wall gradient $\varphi_{\chi}(0)$ exists. The calculation of $\Delta_{c r}^{*}$ is illustrated 


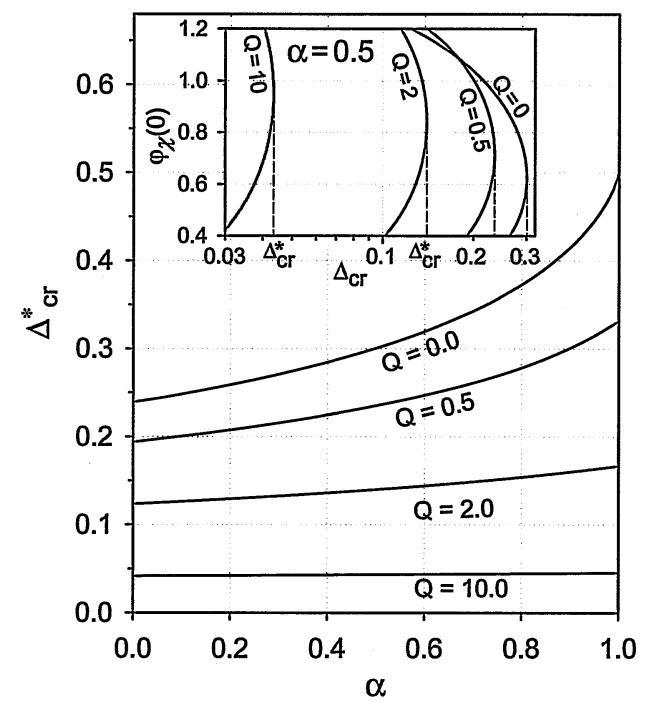

Fig. 12. Computed critical ignition Damköhler numbers $\Delta_{c r}^{*}$ versus $\alpha$ for various values of the parameter $Q$. In the insert, the calculation of $\Delta_{c r}^{*}$ is illustrated from plots of $\varphi_{\chi}(0)$ versus $\Delta_{c r}$ for $\alpha=0.5$ and four values of $Q$.

in the insert of Fig. 12: computed plots of $\varphi_{\chi}(0)$ versus $\Delta_{c r}$ are presented for $\alpha=0.5 ; Q=$ 0 is the infinitely fast surface chemistry solution, and larger $Q$ correspond to slower surface chemistry. Figure 12 provides plots of $\Delta_{c r}^{*}$ versus $\alpha$ constructed with the procedure shown in the insert of Fig. 12. At sufficiently large values of $Q$, Eq. A15 reduces (for $n_{F}=$ 1) to $d^{2} \varphi / d \chi^{2}=-\Delta_{c r} Q \exp (\varphi-\chi)$ : its solution $\Delta_{c r}^{*}\left(\zeta ; D a_{s}\right)$ is such that at large $Q$ the product $\Delta_{c r}^{*}\left(\zeta ; D a_{s}\right) Q$ becomes independent of $\alpha$ or $Q$ and approaches the constant value 0.5 . As shown in Fig. 12, already at $Q=10$, the product $\Delta_{c r}^{*}\left(\zeta ; D a_{s}\right) Q$ ranges from 0.42 to 0.45 . Over the entire range of $\zeta, T_{W} / T_{I N}, P r$, and $L e$ parameter variation, $\Delta_{c r}^{*}\left(\zeta ; D a_{s}\right)$ decreases faster with decreasing $D a_{s}$ than the function $\left[1+H\left(\zeta ; D a_{s}\right)\right]$ increases with decreasing $D a_{s}$; in addition, the term $\left[1+H\left(\zeta ; D a_{s}\right)\right] \Delta_{c r}^{*}\left(\zeta ; D a_{s}\right)$ is always less than unity and the term $[1+$ $\left.H\left(\zeta ; D a_{s}\right)\right] \Delta_{c r}^{*}\left(\zeta ; D a_{s}\right) /\left[\Delta_{c r}^{*}(\zeta)\right]_{D a_{s} \rightarrow \infty}$ is less or equal to unity. Considering, for example, a typical for hydrocarbons value of $\epsilon=0.1$ and using Eqs. 28 and 30, Eq. A11 yields a minimum $D a_{s} \approx 1.0$ required to achieve $Q=10$, which corresponds to $\Delta_{c r}^{*}\left(\zeta ; D a_{s}\right) \approx 0.045$ (see Fig. $12)$; under these conditions Eq. 42 gives $H(\zeta$; $\left.D a_{s}\right)=1.3$ and hence $\left[1+H\left(\zeta ; D a_{s}\right)\right] \Delta_{c r}^{*}(\zeta$; $\left.D a_{s}\right) \approx 0.1$. Furthermore, for the minimum possible value of $\left[\Delta_{c r}^{*}(\zeta)\right]_{D a_{s} \rightarrow \infty} \approx 0.24$ (see Fig. 12), $\left[1+H\left(\zeta ; D a_{s}\right)\right] \Delta_{c r}^{*}\left(\zeta ; D a_{s}\right) /$ $\left[\Delta_{c r}^{*}(\zeta)\right]_{D a_{s} \rightarrow \infty} \approx 0.4$. 